\title{
Discovery of VHE gamma rays from PKS 2005-489
}

F. Aharonian ${ }^{1}$, A. G. Akhperjanian ${ }^{2}$, K.-M. Aye ${ }^{3}$, A. R. Bazer-Bachi ${ }^{4}$, M. Beilicke ${ }^{5}$, W. Benbow ${ }^{1}$, D. Berge ${ }^{1}$, P. Berghaus ${ }^{6, \star}$, K. Bernlöhr ${ }^{1,7}$, C. Boisson ${ }^{8}$, O. Bolz ${ }^{1}$, I. Braun ${ }^{1}$, F. Breitling ${ }^{7}$, A. M. Brown ${ }^{3}$, J. Bussons Gordo ${ }^{9}$, P. M. Chadwick ${ }^{3}$, L.-M. Chounet ${ }^{10}$, R. Cornils ${ }^{5}$, L. Costamante ${ }^{1,20}$, B. Degrange ${ }^{10}$, A. Djannati-Atai ${ }^{6}$, L. O'C. Drury ${ }^{11}$, G. Dubus ${ }^{10}$, D. Emmanoulopoulos ${ }^{12}$, P. Espigat ${ }^{6}$, F. Feinstein ${ }^{9}$, P. Fleury ${ }^{10}$, G. Fontaine ${ }^{10}$, Y. Fuchs ${ }^{13}$, S. Funk ${ }^{1}$, Y. A. Gallant ${ }^{9}$, B. Giebels ${ }^{10}$, S. Gillessen ${ }^{1}$, J. F. Glicenstein ${ }^{14}$, P. Goret ${ }^{14}$, C. Hadjichristidis ${ }^{3}$, M. Hauser ${ }^{12}$, G. Heinzelmann ${ }^{5}$, G. Henri ${ }^{13}$, G. Hermann ${ }^{1}$, J. A. Hinton ${ }^{1}$, W. Hofmann ${ }^{1}$, M. Holleran ${ }^{15}$, D. Horns ${ }^{1}$, O. C. de Jager ${ }^{15}$, B. Khélifi ${ }^{1}$, Nu. Komin ${ }^{7}$, A. Konopelko ${ }^{1,7}$, I. J. Latham ${ }^{3}$, R. Le Gallou ${ }^{3}$, A. Lemière 6 ,

M. Lemoine-Goumard ${ }^{10}$, N. Leroy ${ }^{10}$, T. Lohse ${ }^{7}$, O. Martineau-Huynh ${ }^{16}$, A. Marcowith ${ }^{4}$, C. Masterson ${ }^{1,20}$, T. J. L. McComb ${ }^{3}$, M. de Naurois ${ }^{16}$, S.J. Nolan ${ }^{3}$, A. Noutsos ${ }^{3}$, K. J. Orford ${ }^{3}$, J. L. Osborne ${ }^{3}$, M. Ouchrif ${ }^{16,20}$, M. Panter ${ }^{1}$, G. Pelletier ${ }^{13}$, S. Pita ${ }^{6}$, G. Pühlhofer ${ }^{1,12}$, M. Punch $^{6}$, B. C. Raubenheimer ${ }^{15}$, M. Raue ${ }^{5}$, J. Raux ${ }^{16}$, S. M. Rayner ${ }^{3}$, I. Redondo ${ }^{10,20, \star \star}$, A. Reimer ${ }^{17}$, O. Reimer ${ }^{17}$, J. Ripken ${ }^{5}$, L. Rob ${ }^{18}$, L. Rolland ${ }^{16}$, G. Rowell ${ }^{1}$, V. Sahakian ${ }^{2}$, L. Saugé ${ }^{13}$, S. Schlenker ${ }^{7}$, R. Schlickeiser ${ }^{17}$, C. Schuster ${ }^{17}$, U. Schwanke ${ }^{7}$, M. Siewert ${ }^{17}$, H. Sol $^{8}$, R. Steenkamp ${ }^{19}$, C. Stegmann ${ }^{7}$, J.-P. Tavernet ${ }^{16}$, R. Terrier ${ }^{6}$, C. G. Théoret ${ }^{6}$, M. Tluczykont ${ }^{10,20}$, G. Vasileiadis ${ }^{9}$, C. Venter ${ }^{15}$, P. Vincent ${ }^{16}$, H. J. Völk ${ }^{1}$, and S. J. Wagner ${ }^{12}$

\begin{abstract}
${ }^{1}$ Max-Planck-Institut für Kernphysik, Heidelberg, Germany, e-mail: Wystan. Benbow@mpi-hd.mpg.de; ${ }^{2}$ Yerevan Physics Institute, Armenia; ${ }^{3}$ University of Durham, Department of Physics, UK; ${ }^{4}$ Centre d'Étude Spatiale des Rayonnements, CNRS/UPS, Toulouse, France; ${ }^{5}$ Universität Hamburg, Institut für Experimentalphysik, Germany; 6 APC, Paris, France $^{\star \star \star} ;{ }^{7}$ Institut für Physik, Humboldt-Universität zu Berlin, Germany; ${ }^{8}$ LUTH, UMR 8102 du CNRS, Observatoire de Paris, Section de Meudon, France; ${ }^{9}$ Groupe d'Astroparticules de Montpellier, IN2P3/CNRS, Université Montpellier II, France; ${ }^{10}$ Laboratoire Leprince-Ringuet, IN2P3/CNRS, École Polytechnique, Palaiseau, France; ${ }^{11}$ Dublin Institute for Advanced Studies, Ireland; ${ }^{12}$ Landessternwarte, Königstuhl, Heidelberg, Germany; ${ }^{13}$ Laboratoire d'Astrophysique de Grenoble, INSU/CNRS, Université Joseph Fourier, France; ${ }^{14}$ DAPNIA/DSM/CEA, CE Saclay, Gif-sur-Yvette, France; ${ }^{15}$ Unit for Space Physics, North-West University, Potchefstroom, South Africa; ${ }^{16}$ Laboratoire de Physique Nucléaire et de Hautes Énergies, IN2P3/CNRS, Universités Paris VI \& VII, France; ${ }^{17}$ Institut für Theoretische Physik, Lehrstuhl IV, RuhrUniversität Bochum, Germany; ${ }^{18}$ Institute of Particle and Nuclear Physics, Charles University, Prague, Czech Republic; ${ }^{19}$ University of Namibia, Windhoek, Namibia; ${ }^{20}$ European Associated Laboratory for Gamma-Ray Astronomy, jointly supported by CNRS and MPG
\end{abstract}

Received 29 March 2005 / Accepted 24 April 2005

\begin{abstract}
The high-frequency peaked BL Lac PKS 2005-489 $(z=0.071)$ was observed in 2003 and 2004 with the HESS stereoscopic array of imaging atmospheric-Cherenkov telescopes in Namibia. A signal was detected at the $6.7 \sigma$ level in the 2004 observations ( $24.2 \mathrm{~h}$ live time), but not in the 2003 data set ( $27.3 \mathrm{~h}$ live time). PKS $2005-489$ is the first blazar independently discovered by HESS to be an emitter of VHE photons, and only the second such blazar in the Southern Hemisphere. The integral flux above $200 \mathrm{GeV}$ observed in 2004 is $\left(6.9 \pm 1.0_{\text {stat }} \pm 1.4_{\text {syst }}\right) \times 10^{-12} \mathrm{~cm}^{-2} \mathrm{~s}^{-1}$, corresponding to $\sim 2.5 \%$ of the flux observed from the Crab Nebula. The $99 \%$ upper limit on the flux in $2003, I(>200 \mathrm{GeV})<5.2 \times 10^{-12} \mathrm{~cm}^{-2} \mathrm{~s}^{-1}$, is smaller than the flux measured in 2004, suggesting an increased level of activity in 2004. However, the data show no evidence for significant variability on any time scale less than a year. An energy spectrum is measured and is characterized by a very soft power law (photon index of $\Gamma=4.0 \pm 0.4$ ).
\end{abstract}

Key words. galaxies: active - BL Lacertae objects: individual: PKS 2005-489 - gamma rays: observations

\section{Introduction}

^ Université Libre de Bruxelles, Faculté des Sciences, Belgium.

$\star \star$ Now at Department of Physics and Astronomy, Univ. of Sheffield, UK.

$\star \star \star$ UMR 7164 (CNRS, Université Paris VII, CEA, Observatoire de Paris).
PKS 2005-489 was initially discovered as a bright ( $>0.5 \mathrm{Jy})$ radio source at $2.7 \mathrm{GHz}$ (Wall et al. 1975) and later identified as a very bright BL Lac object (Wall et al. 1986). It is classified as a high-frequency peaked BL Lac (HBL) due to its X-ray-to-radio flux ratio (Sambruna et al. 1995) and 
Table 1. Shown are the configurations of HESS with which PKS 2005-489 was observed, the number of observation runs, the dead time corrected observation time, the mean zenith angle of the observations $\left(Z_{\mathrm{obs}}\right)$, the post-cuts energy threshold at $Z_{\mathrm{obs}}$, the number of on-source and off-source events passing the cuts, the normalization for the off-source events, the observed excess from PKS 2005-489, and the significance of the excess.

\begin{tabular}{|c|c|c|c|c|c|c|c|c|c|c|c|}
\hline $\begin{array}{c}\text { Dark } \\
\text { periods }\end{array}$ & Configuration & $N_{\text {tel }}$ & $N_{\text {runs }}$ & $\begin{array}{c}\text { Obs. Time } \\
{[\mathrm{h}]}\end{array}$ & $\begin{array}{c}Z_{\mathrm{obs}} \\
{\left[{ }^{\circ}\right]}\end{array}$ & $\begin{array}{c}E_{\mathrm{th}} \\
{[\mathrm{GeV}]}\end{array}$ & On & Off & Norm & Excess & $\begin{array}{l}\text { Sig. } \\
{[\sigma]}\end{array}$ \\
\hline $6 / 2003$ & Offline Stereo & 2 & 22 & 7.9 & 28 & 340 & 194 & 1177 & 0.1567 & 10 & 0.7 \\
\hline 7 and $8 / 2003$ & Online Stereo & 2 & 43 & 18.6 & 29 & 250 & 628 & 3717 & 0.1585 & 39 & 1.5 \\
\hline $9 / 2003$ & Online Stereo & 3 & 2 & 0.8 & 26 & 240 & 56 & 384 & 0.1574 & -4 & -0.5 \\
\hline $6,7,9$ and $10 / 2004$ & Online Stereo & 4 & 57 & 24.2 & 38 & 300 & 1762 & 9410 & 0.1567 & 288 & 6.7 \\
\hline Total & & & & & & & & & & & 6.3 \\
\hline
\end{tabular}

because its broadband spectral energy distribution (SED) peaks in the UV. It has been the target of several multi-wavelength observation campaigns and is well studied from the radio to the X-ray regime. PKS 2005-489 was also marginally detected by EGRET at energies greater than $100 \mathrm{MeV}$ (Lin et al. 1996) and in the $\mathrm{GeV}$ regime (Lamb \& Macomb 1997). It is among the closest $(z=0.071)$ Southern Hemisphere HBLs (Falomo et al. 1987). Based on its SED and its proximity, PKS 2005-489 is viewed as a promising candidate for detection as a VHE emitter (Costamante \& Ghisellini 2002; Perlman 1999; Stecker et al. 1996). However, it has not been previously detected in the VHE regime. The CANGAROO collaboration reported upper limits on the flux above $2 \mathrm{TeV}$ in 1993-1994 (Roberts et al. 1998), above $1.5 \mathrm{TeV}$ in 1997 (Roberts et al. 1999), above $1.1 \mathrm{TeV}$ in 1999 and above $450 \mathrm{GeV}$ in 2000 (Nishijima 2002). The University of Durham group has published the most constraining upper limit $(3 \sigma)$ on the flux, $I(>400 \mathrm{GeV})<7.9 \times 10^{-12} \mathrm{~cm}^{-2} \mathrm{~s}^{-1}$, based on observations made from 1996-1999 with the Mark 6 Telescope (Chadwick et al. 2000). As upper limits are of limited value for interpreting an SED, the present discovery of VHE gamma-rays from PKS 2005-489 yields considerably more insight into the understanding of this object and VHE AGN in general.

\section{HESS detector}

The HESS experiment, located in the Khomas Highlands of Namibia $\left(23^{\circ} 16^{\prime} 18^{\prime \prime} \mathrm{S}, 16^{\circ} 30^{\prime} 1^{\prime \prime} \mathrm{E}, 1835 \mathrm{~m}\right.$ above sea level), is designed to search for astrophysical $\gamma$-ray emission above $\sim 100 \mathrm{GeV}$. The detector consists of a system of four imaging atmospheric-Cherenkov telescopes (diameter $13 \mathrm{~m}$, focal length $15 \mathrm{~m}$, mirror area $107 \mathrm{~m}^{2}$ ) in a square of $120 \mathrm{~m}$ side. Each telescope is equipped with a camera that provides a $5^{\circ}$ field of view (f.o.v.) and contains 960 individual photomultiplier pixels, subtending $0.16^{\circ}$ each, with Winston cone light concentrators. A HESS camera is triggered when one of 38 overlapping 64-pixel sectors has a minimum number of pixels with a signal above a threshold in photoelectrons (PEs) coincident in an effective $\sim 1.3 \mathrm{~ns}$ trigger window. Once a camera has triggered, a signal is sent out to a central trigger system (Funk et al. 2004) which allows for a multiple telescope coincidence requirement (presently a minimum of two triggered telescopes). The sensitivity of HESS ( $5 \sigma$ in $25 \mathrm{~h}$ for a $1 \% \mathrm{Crab}$ Nebula flux source at $20^{\circ}$ zenith angle) allows for detection of VHE emission from objects such as PKS 2005-489 at previously undetectable flux levels. More details on HESS can be found in Bernlöhr et al. (2003), Hofmann (2003), and Vincent et al. (2003).

\section{Observations}

The HESS observations of PKS 2005-489 in 2003 were made while the system was under construction. Therefore the data were obtained using different instrument configurations. Most observations in 2003 were made using a two-telescope array, with the exception of a small amount of data taken after the addition of the third telescope to the array in September 2003. Another variation arises because the HESS central trigger system was installed in July 2003. Before this time twotelescope data were taken with each telescope separately, and the stereo multiplicity requirement was performed off-line ("Offline Stereo") using GPS time stamps. After the installation of the central trigger system, the stereo multiplicity requirement was performed in the hardware ("Online Stereo"). As the central trigger system reduced the recording rate considerably, the camera trigger threshold was lowered (3 pixels $>5.3$ PEs vs. 4 pixels $>6.7$ PEs). This increased the rates, while maintaining a reduced system dead time, and ultimately resulted in a lower energy threshold. All observations made in 2004 use the full four-telescope array.

Table 1 gives details of the observations of PKS 2005-489 by HESS which pass selection criteria which remove data for which the weather conditions were poor or the hardware was not functioning properly. The data were taken in $28 \mathrm{~min}$ runs using Wobble mode, i.e. the source direction is positioned $\pm 0.5^{\circ}$ relative to the center of the f.o.v. of the camera during observations, which allows for both on-source observations and simultaneous estimation of the background induced by charged cosmic rays.

\section{Analysis technique}

The analysis of the data passing the run selection criteria proceeds in the following steps: first the images are calibrated (Aharonian et al. 2004) and then "cleaned" to remove night sky 
Table 2. The selection cuts applied to the data. The same cuts are used, regardless of the number of telescopes, for all Online Stereo configurations.

\begin{tabular}{cccccc}
\hline \hline Stereo & MRSL & MRSL & MRSW & MRSW & $\theta^{2}$ \\
Config. & $\min$ & $\max$ & $\min$ & $\max$ & \\
& {$[\sigma]$} & {$[\sigma]$} & {$[\sigma]$} & {$[\sigma]$} & {$\left[\mathrm{deg}^{2}\right]$} \\
\hline Offline & 1.3 & -2.2 & 1.1 & -2.5 & 0.02 \\
Online & 2.0 & -2.0 & 0.9 & -2.0 & 0.0125 \\
\hline
\end{tabular}

background noise from the image. The cleaning is done using a two-stage tail-cut procedure (thresholds: 5 and 10 PEs). The moments of the shower image are then parameterized using a Hillas-type analysis (Hillas 1985), and the shower geometry is reconstructed using the intersection of image axes, giving a typical angular resolution of $\sim 0.1^{\circ}$ per event and an average accuracy of $\sim 10 \mathrm{~m}$ in the determination of the shower core location. Only images which exceed a minimum total signal (80 PEs for Online Stereo, 90 PEs for Offline Stereo) and which pass a distance cut requiring the image center of gravity to be less than $2^{\circ}$ from the center of the camera are used in the reconstruction. The size cut (minimum total signal) ensures that the images are well reconstructed and the distance cut eliminates effects from truncation of images by the edge of the camera.

After the event reconstruction, the much more numerous cosmic-ray background events are rejected using cuts on mean reduced scaled width (MRSW) and length (MRSL) parameters (Aharonian et al. 2005). A cut on $\theta^{2}$, the square of the angular difference between the reconstructed shower position and the source position, is also applied. All the cuts (shown in Table 2) are optimized a priori (simultaneously) using Monte Carlo gamma-ray simulations and off-source data to yield the maximum expected significance per hour of observation for a weak source. However, the significance expected (and observed) is not strongly dependent on the exact values of the cuts.

The background is estimated using all events passing cuts in a ring (central radius $0.5^{\circ}$ ) around the source location. The ring width is chosen such that the area of the ring is approximately seven times the area of the on-source region (i.e. the area, centered on the source location, falling within the $\theta^{2}$ cut). The relative statistical error on the background measurement is reduced by the use of a larger background region. A small correction accounting for the radial acceptance of the camera is applied to the normalization of the off-source event total. The significance of any excess is calculated following the method of Eq. (17) in Li \& Ma (1983).

\section{Results}

Table 1 shows the results of the HESS observations for each of the detector configurations. A significant excess of events in the direction of PKS 2005-489 is detected in $2004(6.7 \sigma)$, but not in $2003(1.4 \sigma)$. The total significance of the excess for all observations is $6.3 \sigma$. Figure 1 shows the on-source and normalized off-source distributions of $\theta^{2}$ for all observations in 2004.

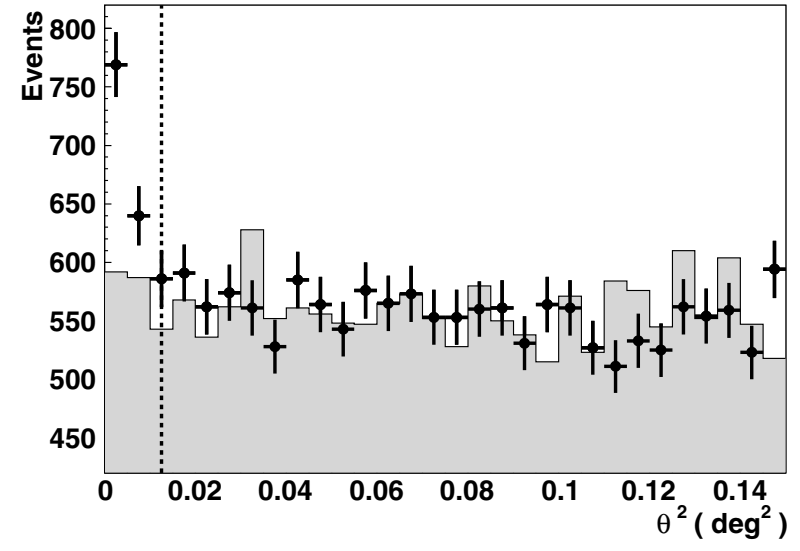

Fig. 1. The distribution of $\theta^{2}$ for on-source events (points) and normalized off-source events (shaded) from observations of PKS 2005-489 in 2004. The dashed line represents the cut on $\theta^{2}$ applied to the data.

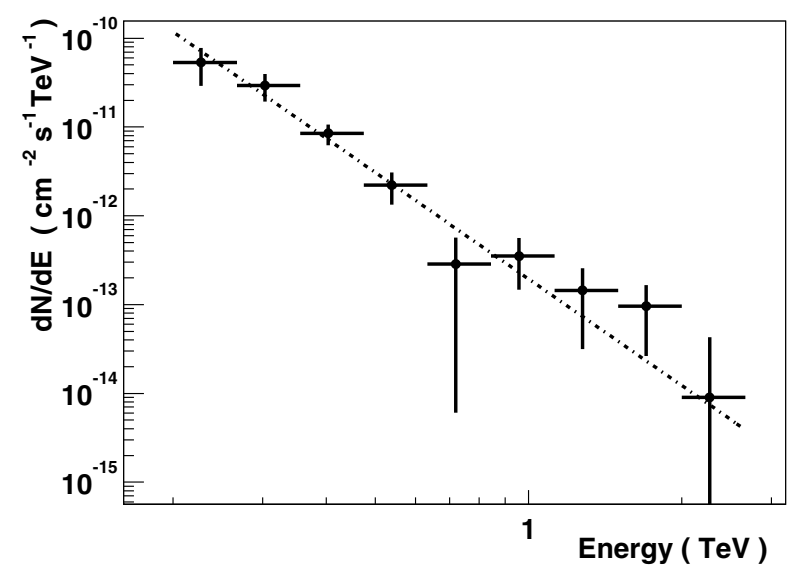

Fig. 2. The energy spectrum of HESS J2009-488. The dashed line represents the best $\chi^{2}$ fit of a power law.

The background is flat in $\theta^{2}$ as expected, and there is a clear excess at small values of $\theta^{2}$ corresponding to the observed signal. A two-dimensional fit of the excess observed finds the shape to be characteristic of a point source, located (J2000) at ( $\alpha=$ $\left.20^{\mathrm{h}} 9^{\mathrm{m}} 29.3^{\mathrm{s}} \pm 2.7_{\text {stat }}^{\mathrm{s}} \pm 1.3_{\text {syst }}^{\mathrm{s}}, \delta=-48^{\circ} 49^{\prime} 19^{\prime \prime} \pm 36_{\text {stat }}^{\prime \prime} \pm 20_{\text {syst }}^{\prime \prime}\right)$. The excess, named HESS J2009-488, is consistent with the position of the blazar $\left(\alpha=20^{\mathrm{h}} 9^{\mathrm{m}} 25.4^{\mathrm{s}}, \delta=-48^{\circ} 49^{\prime} 53.7^{\prime \prime}\right)$ as expected, and is therefore assumed to be associated with PKS 2005-489.

As the energy resolution of HESS is $\sim 15 \%$ per event, accurate energy spectra can be measured. The energy spectrum for the 2004 data set is shown in Fig. 2. The best $\chi^{2}$ fit of a power law $\left(\mathrm{d} N / \mathrm{d} E \sim E^{-\Gamma}\right)$ to these data yields a photon index $\Gamma=4.0 \pm 0.4_{\text {stat }}$, and a $\chi^{2}$ of 5.6 for 7 degrees of freedom. The systematic error on the photon index is small compared to the statistical error. It should be noted that each of the five highest energy points, $E>0.64 \mathrm{TeV}$, in Fig. 2 have statistical significance less than $2 \sigma$. However, removing these points from the fit does not change $\Gamma$ significantly. Consistent results are also found using alternative background estimation techniques and/or independent analysis chains. No evidence is found for significant features, such as a cutoff or break, in the energy spectrum. 
Assuming the determined photon index of $\Gamma=4.0$, the integral flux above $200 \mathrm{GeV}$ measured in 2004 is $I(>200 \mathrm{GeV})=$ $\left(6.9 \pm 1.0_{\text {stat }} \pm 1.4_{\text {syst }}\right) \times 10^{-12} \mathrm{~cm}^{-2} \mathrm{~s}^{-1}$. This corresponds to $\sim 2.5 \%$ of $I(>200 \mathrm{GeV})$ determined by HESS from the Crab Nebula. The $99 \%$ confidence limit (Feldman \& Cousins 1998) on $I(>200 \mathrm{GeV})$ in 2003 is $5.2 \times 10^{-12} \mathrm{~cm}^{-2} \mathrm{~s}^{-1}$. This is slightly less than the flux in 2004 suggesting that PKS 2005-489 was in a higher state during the 2004 observations. The flux upper limit in 2003 and the flux in 2004 are well below all previously published upper limits for this object. No evidence for variability in 2003 or 2004 is found as fits to the integral flux versus time are consistent with being constant. This is the case whether the data are binned by dark period (months) within each year $\left(\chi^{2}\right.$ probability, $P\left(\chi^{2}\right)$, of 0.57 in 2003 , and 0.98 in 2004), by nights within each dark period $\left(P\left(\chi^{2}\right)>0.2\right.$ and 0.6 for each of the four periods in 2003 and 2004, respectively) or runs $(\sim 30 \mathrm{~min})$ within individual nights $\left(P\left(\chi^{2}\right)>\right.$ 0.05 and 0.1 for all nights in 2003 (mean $P\left(\chi^{2}\right)=0.45$ ) and 2004 (mean $P\left(\chi^{2}\right)=0.53$ ), respectively). However, given the low statistics overall, the lack of observed short time scale variability in 2003 and 2004 is not surprising.

\section{Conclusions}

PKS 2005-489 has been detected by HESS at energies greater than $200 \mathrm{GeV}$ in 2004. It is the first AGN independently discovered by HESS as an emitter of VHE photons and only the second such AGN known in the Southern Hemisphere. The measured VHE flux is quite low $(\sim 2.5 \%$ of the Crab Nebula flux) and no evidence supporting variability of the VHE flux on time scales of less than a year is found. However, the upper limit resulting from the lack of a detection in 2003 suggests that the flux from PKS 2005-489 in 2004 was higher than the previous year. This inference is supported by the behavior of this blazar in the X-ray regime. Quick-look results provided by the ASM/RXTE team show the average count rate from PKS 2005-489 was a factor of $\sim 3$ higher in 2004 (0.116 \pm $\left.0.025 \mathrm{~s}^{-1}\right)$ than in $2003\left(0.039 \pm 0.026 \mathrm{~s}^{-1}\right)$. Interestingly, the average ASM count rate in $1998\left(0.39 \pm 0.02 \mathrm{~s}^{-1}\right)$ is considerably higher than that in 2004, suggesting that PKS 2005-489 was in a low state during the HESS observations. Should the VHE flux increase comparably to the historical (1998 vs. 2004) $\mathrm{X}$-ray count rate, a significant signal will quickly accumulate $(\sim 1 \mathrm{~h})$ in HESS observations allowing for more detailed studies of the VHE behavior to be performed.

The VHE spectrum of PKS 2005-489 is the softest $(\Gamma=$ 4.0) ever measured from a BL Lac. Given the proximity $(z=$ 0.071) of PKS 2005-489, the softness is unlikely to be largely due to absorption of VHE photons on the extragalactic background light. Assuming the softness to be intrinsic to the blazar, inverse-Compton models of the VHE emission predict that the $\mathrm{X}$-ray spectrum should also be steep. A multi-wavelength observation campaign (including X-ray energies) was performed in October 2004, results of which will address this issue among others, but is beyond the scope of this letter.
Given its low flux and soft spectrum, PKS 2005-489 was not detectable by previous generations of VHE instruments. The currently unprecedented ability of HESS to detect faint soft-spectrum sources of VHE gamma-rays, such as AGN, should significantly improve the overall understanding of blazars and their physics.

Acknowledgements. The support of the Namibian authorities and of the University of Namibia in facilitating the construction and operation of HESS is gratefully acknowledged, as is the support by the German Ministry for Education and Research (BMBF), the Max-Planck-Society, the French Ministry for Research, the CNRS-IN2P3 and the Astroparticle Interdisciplinary Programme of the CNRS, the U.K. Particle Physics and Astronomy Research Council (PPARC), the IPNP of the Charles University, the South African Department of Science and Technology and National Research Foundation, and by the University of Namibia. We appreciate the excellent work of the technical support staff in Berlin, Durham, Hamburg, Heidelberg, Palaiseau, Paris, Saclay, and in Namibia in the construction and operation of the equipment.

\section{References}

Aharonian, F., Akhperjanian, A. G., Aye, K.-M., et al. 2004, Astropart. Phys., 22, 109

Aharonian, F., Akhperjanian, A. G., Aye, K.-M., et al. 2005, A\&A, 430,865

Bernlöhr, K., Carrol, O., Cornils, R., et al. 2003, Astropart. Phys., 20, 111

Chadwick, P. M., Daniel, M. K., Lyons, K., et al. 2000, A\&A, 364, 450

Costamante, L., \& Ghisellini, G. 2002, A\&A, 384, 56

Falomo, R., Maraschi, L., Treves, A., et al. 1987, ApJ, 318, L39

Feldman, G. J., \& Cousins, R. D. 1998, Phys. Rev. D, 57, 3873

Funk, S., Hermann, G., Hinton, J., et al. 2004, Astropart. Phys., 22, 285

Hillas, A. 1985, Proc. of the 19th ICRC (La Jolla), 3, 445

Hofmann, W. 2003, Proc. of the 28th ICRC (Tsukuba), 2811

Lamb, R. C., \& Macomb, D. J. 1997, ApJ, 488, 872

Li, T., \& Ma, Y. 1983, ApJ, 272, 317

Lin, Y. C., Bertsch, D. L., Dingus, B. L., et al. 1996, ApJS, 105, 331

Nishijima, K. 2002, Publ. Astron. Soc. Aust., 19, 26

Perlman, E. S. 1999, AIP Conf. Proc., 515, 56

Roberts, M. D., Dazeley, S. A., Edwards, P. G., et al. 1998, A\&A, 337, 25

Roberts, M. D., McGee, P., Dazeley, S. A., et al. 1999, A\&A, 343, 691

Sambruna, R. M., Urry, C. M., Ghisellini, G., et al. 1995, ApJ, 449, 567

Stecker, F. W., de Jager, O. C., \& Salamon, M. H. 1996, ApJ, 473, L75

Vincent, P., Denance, J.-P., Huppert, J.-F., et al. 2003, Proc. of the 28th ICRC (Tsukuba), 2887

Wall, J. V., Danzinger, I. J., Pettini, I., et al. 1986, MNRAS, 219, 23

Wall, J. V., Shimmins, A. J., \& Bolton, J. G. 1975, Aust. J. Phys. Astrophys. Suppl., 34, 55 